\title{
Flow structure in a flat channel with honeycomb surfaces
}

\author{
Victor Terekhov ${ }^{1,2}$, Jaroslav Smulsky ${ }^{1}$, Konstantin Sharov ${ }^{1}$, and Alexey Zolotukhin ${ }^{*}, 1,2$ \\ ${ }^{1}$ Institute of Thermophysics SS Kutateladze SB RAS, 630090, Novosibirsk, 1 Ac.. Lavrentiev Ave., \\ Russia \\ ${ }^{2}$ Novosibirsk State Technical University, 630073, Novosibirsk, 20 Karl Marks Avenue, Russia
}

\begin{abstract}
This paper deals with the experimental study of the gas flow structure at longitudinal flow around a honeycomb surface with hexagonal cells of various sizes and depths. The measurements were carried out using the PIV system for the developed flow in a flat channel with the crosssection $21 \times 150 \mathrm{~mm}$ and length of $1000 \mathrm{~mm}$. The flow was visualized and the velocity and turbulence components were measured in a smooth channel and in the presence of honeycomb surfaces. It is shown that a boundary layer with high gradient of velocity and turbulent pulsations is formed near the honeycomb.
\end{abstract}

\section{Introduction}

An important direction in the development of the heat transfer theory and aerohydrodynamics is the search for new methods of heat transfer intensification with a parity growth of hydraulic losses. In this respect, the surfaces with lunar reliefs are illustrative $[1,2]$. A number of experimental and numerical studies in this field prove a great potential of such methods.

The honeycomb surfaces considered in this work, used in particular for the purposes of thermal protection of spacecraft elements and as seals for gas turbines, have a number of similarities to the cavity vortex generators. This is evidenced by the results of the first experimental studies of the longitudinal flow around the surfaces with honeycomb coatings $[3,4]$. Data on acoustic damping [5], which helps to reduce friction, give all the grounds for using such vortex generators as the effective methods for controlling the processes of turbulent transfer. However, due to the lack of detailed experimental information on the flow structure, it is not possible to create an integrated pattern of this complex phenomenon, and therefore its deeper investigation is required.

The development of the flow field and turbulent characteristics along the honeycomb surfaces are studied in this paper. It compares data on the averaged and turbulent characteristics for the honeycombs and channel with smooth walls.

\footnotetext{
* Corresponding author: zoloav@mail.ru
} 


\section{Experimental setup and measurement methods}

The working channel of the experimental setup had a rectangular section of $21 \times 150 \mathrm{~mm}$, length of $1 \mathrm{~m}$, and height $\mathrm{h}=22 \mathrm{~mm}$. It was made of transparent organic glass, $8 \mathrm{~mm}$ thick. The transverse dimensions of the inlet channel were chosen to ensure the two-dimensional flow in the central longitudinal cross-section of the channel. At the distance of $580 \mathrm{~mm}$ from the channel inlet, the plates were mounted flush with the following dimensions: the first plate of $40 \times 200 \mathrm{~mm}$, and the second and third plates of $58.4 \times 182 \mathrm{~mm}$ with hexagonal cells. The cells were blocked from the outside. Their heights $\mathrm{b}$ were $21 \mathrm{~mm}, 2.4$ $\mathrm{mm}$, and $6 \mathrm{~mm}$. The first honeycomb surface was made of textolite with the thickness of cell partitions of $0.2 \mathrm{~mm}$ and diameter of a circle circumscribed around a cell of $5 \mathrm{~mm}$. Honeycombs No. 2 and No. 3 were made of plastic and had the cell diameter of $12 \mathrm{~mm}$ and wall thickness of $0.5 \mathrm{~mm}$. The cell diameter-height ratio $\mathrm{d} / \mathrm{b}$ was 0.24 for the first plate, and for the second and third plates, it was 2 and 5, respectively.

The experimental setup includes all elements of the wind tunnel to form a qualitative flow: prechamber, nozzle and working channel. The detailed description of setup and measurement system is given in [6]. In the present experiments, development of the flow along the entire length of the honeycomb plate was studied at three different velocities: 5 , 10 and $20 \mathrm{~m} / \mathrm{s}$; in the beginning of the plate with honeycombs, the flow was developed. The measurements were performed using the PIV system.

\section{Experimental results and discussion}

The paper presents the results of measurements of longitudinal velocity profiles, longitudinal and transverse pulsations, and shear stresses in a smooth channel and channel with honeycombs.
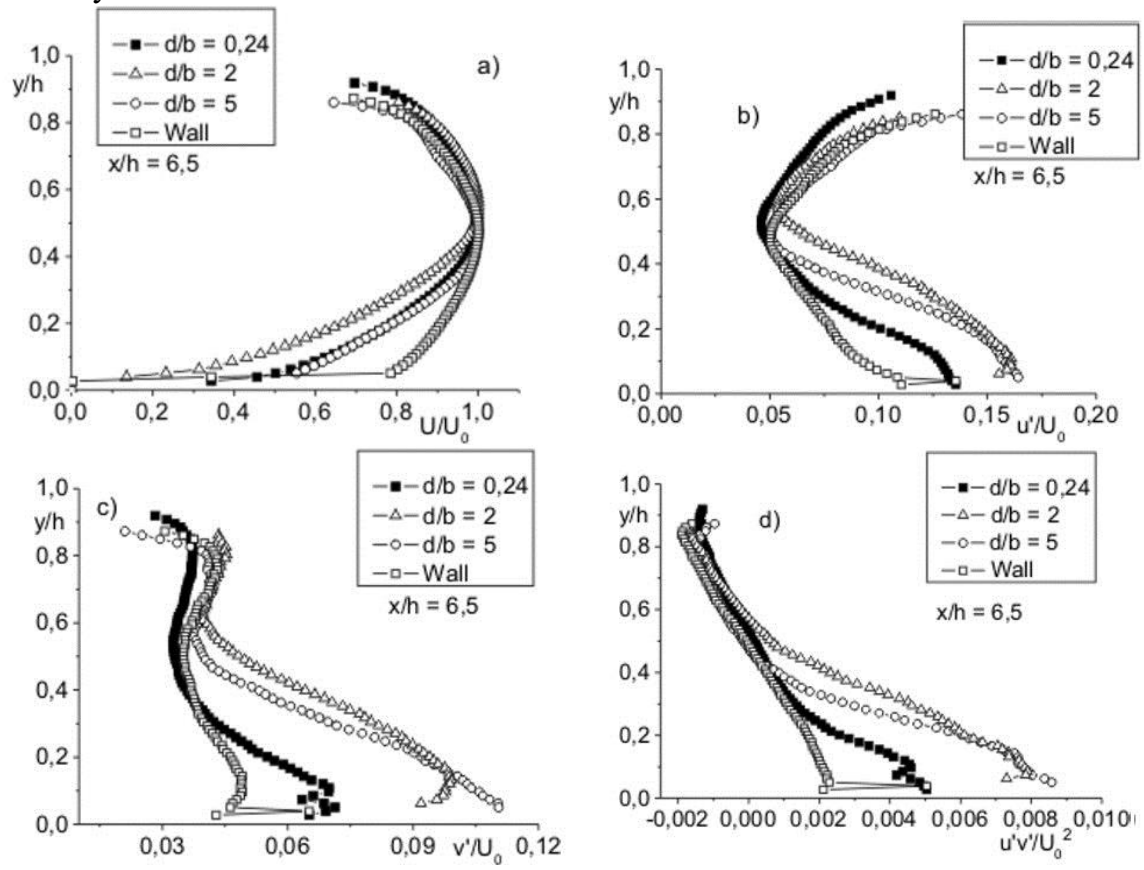

Fig. 1. Distribution along the channel height: a) longitudinal velocity, b) longitudinal, c) transverse fluctuations, and d) shear stresses, working fluid - air, $\operatorname{Re}=6,4 \cdot 10^{4}$ 
All data were obtained for cross-section at $\mathrm{x} / \mathrm{h}=6.5, \mathrm{x}$ is a distance from the beginning of the surface with hexagonal cells. According to experiments, the flow in a vicinity of the honeycomb surface is fundamentally different from the flow around a smooth wall. In the near-wall region of the honeycomb surface, the velocity profile is more even in comparison with the classical distribution on a smooth wall (Fig. 1a). At the same time, longitudinal and transverse velocity pulsations (Figs. $1 \mathrm{~b}$ and 1c, respectively) near the honeycomb surface are several times greater than on the smooth surface. A similar tendency exists for distribution of shear stresses across the channel (Fig. 1d). If for the channel with smooth walls, as expected, there is the linear distribution of shear stresses along the height, in the channel with cells at distance $\mathrm{y} / \mathrm{h} \sim 0.2$, the stresses increase more than 2-3 times. At the same time, a steady tendency to a decrease in transverse velocity pulsations and turbulent shear stresses is observed directly in the near-wall region.

Such a complex scenario of flow development is caused by interaction of the boundary layer with the honeycomb cells. Indeed, the flow around depressions of various shapes (cylindrical cavity [7], hole [8], transverse trench-groove [9], etc.) on the surface leads to formation of a periodic vortex layer behind them. However, we failed to detect the existence of such structures in the present work.

One of the principal differences between the obtained results and experimental data of $[3,4]$ is the absence of the flow slip along the surface with honeycombs, which, according to these data, could reach about $20-30 \%$ of the velocity in the flow core.

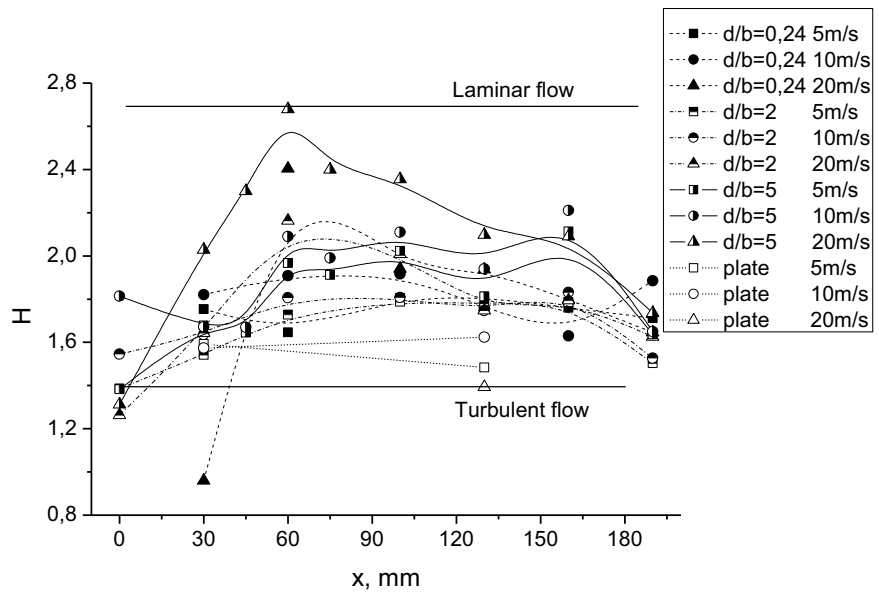

Fig. 2. Change in form parameter $\mathrm{H}$ along the honeycomb surface.

Distribution of layer form parameter $\mathrm{H}=\delta^{*} / \delta^{* *}$ along the honeycombs with different ratio $\mathrm{d} / \mathrm{b}$ is shown in Fig. 2 for three flow velocities (coordinate $x=0$ corresponds to the beginning of honeycomb region).

In the diagram, the value $\mathrm{H}$ corresponds to the developed turbulent flow; then, we can observe its increase to the value characteristic of the laminar flow regime $(\mathrm{H} \rightarrow 2.7)$ and subsequent decrease. This behavior of the form parameter is caused, most likely, by the "flattening" of velocity profile with simultaneous increase in turbulence and tangential stresses.

It is not possible now to formulate clearly the reasons for such a behavior of local and integral characteristics of the turbulent flow around a surface with honeycombs, primarily because of multifactority and complexity of the aerodynamic structure, and this will require further detailed studies. 


\section{Conclusions}

The paper presents the results of experimental study of the averaged flow, normal and tangential stresses in gas flowing with different velocities around the honeycomb surfaces with characteristic cell size of $\sim 5 \mathrm{~mm}$ and $\sim 12 \mathrm{~mm}$.

The measurements were performed using the two-component PIV system. The flow was organized in a flat channel of $21 \times 150 \times 1000 \mathrm{~mm}$.

It was found that the boundary layer on the surface with hexagonal cells is less filled than on a smooth wall. At the same time, the growth of turbulent pulsations and tangential stresses is observed simultaneously. However, there is no effect of flow sliding relative to the cells.

The work was financially supported by the grant of the President of the Russian Federation for the leading scientific schools (project NSh-8780.2016.8.).

\section{References}

1. S.A. Isaev, N.V. Kornev, A.I. Leontiev, E. Hassel, Int. J. Heat Mass Transfer 53, 178 (2010)

2. G.V. Kovalenko, V.I. Terekhov, A.A. Khalatov, J. Appl. Mech. Tech. Phys. 51, 839 (2010)

3. A.A. Klimov, S.A. Trdat'yan, High Temperature, 41, 801 (2003)

4. S.A. Trdatyan, A.A. Klimov, Proc. 12-th Int. Heat Transfer Conf. Grenoble. 221. (2002)

5. N.N. Koval'nogov, Energy Problem, № 5-6, 41, (2003) [in Russian]

6. V.I. Terekhov, Ya.I. Smulsky, K.A. Sharov, A.V. Zolotukhin, Thermophys. Aeromech., 21719 (2014)

7. M. Hiwada, T. Kawamura, J. Mabuchi, M. Kumada, Bull. JSME. 26, 1774 (1983)

8. V.I. Terekhov, S.V. Kalinina, J.M. Mshvidobadze, Siberian Phys.-Tech. J. 77 (1992)

9. V.I. Terekhov, N.I. Yarygina, A.V. Shaporin, Industrial Heat Eng. 21, 22 (1999) 